\title{
Arbor
}

\section{Los últimos 20 años de los Centros Sanitarios en España}

\section{Victor Conde Rodelgo}

Arbor CLXX, 670 (Octubre 2001), 247-275 pp.

La lectura de este artículo permite realizar un recorrido por el camino que nuestro sistema sanitario ha efectuado a lo largo de estos últimos años, resaltando los hitos relevantes y los substratos politicos, económicos y sociales que fundamentan decisiones que marcaron la trayectoria de nuestra sanidad.

Con el objeto de comprender mejor la situación de los centros sanitarios en España en los últimos veinte años, es preciso referir aunque sea de forma muy resumida, la situación existente en este sector en la época próxima anterior a la que pretendemos abordar. De forma general y en relación con su adscripción patrimonial, hay que diferenciar los de la Administración Central del Estado, los de las Corporaciones Locales, de la Seguridad Social, entidades benéfico-privadas o instituciones privadas, así como al conjunto de actividades privadas de los médicos y de otro personal sanitario.

Este empeño resulta relativamente fácil cuando lo que se analiza es el equipamiento y la evolución de los centros hospitalarios, pero no sucede lo mismo con los extrahospitalarios que ofrecen una gran variedad en cuanto a su actividad preferente -salud pública, asistencial o de otra naturaleza-, a su dependencia funcional, por su amplia dis- 
tribución territorial, su elevado número, así como por el hecho de no disponer de catálogos o relaciones completas de estas instituciones.

\section{Establecimientos sanitarios dependientes de la Administración Central}

Como establecimientos sanitarios subordinados a la Administración Central, se han contabilizado históricamente los centros adscritos al Ministerio de la Gobernación a través de las Direcciones Generales de Sanidad o de la Beneficencia General del Estado; al Ministerio de Educación representados, esencialmente, por los Hospitales Clínicos de las Facultades de Medicina; al Ministerio de Justicia con las instituciones asistenciales penitenciarias y a los Ministerios militares a través de la Sanidad diferenciada para cada uno de los ejércitos y, posteriormente, integrados en el Ministerio de Defensa.

En el sector público hay que sumar los centros del Seguro Obligatorio de Enfermedad (SOE), bajo la tutela del Ministerio de Trabajo y administrados por el Instituto Nacional de Previsión (INP), y posteriormente por el INSALUD, así como los adscritos a la Obra Sindical «18 de julio», que se integrará, más tarde, en el año 1976, en el referido INP, así como otros tutelados por este Departamento.

Los centros sanitarios del Ministerio de la Gobernación, funcionalmente adscritos a la Dirección General de Sanidad, quedaron encuadrados, todos ellos, en el Organismo autónomo «Administración Institucional de la Sanidad Nacional» (AISNA) de acuerdo con lo previsto en el Decreto-Ley de diciembre de 1972.

NOTA 1. En el Organismo AISNA se integraron los centros del Patronato Nacional Antituberculoso y de las Enfermedades del Tórax, el Patronato Nacional de Asistencia Psiquiátrica, Gran Hospital de la Beneficencia General del Estado, Hospital del Niño Jesús, Instituto Oftálmico Nacional, Hospital Nacional de Enfermedades Infecciosas, Instituto Nacional de Oncología, Instituto Leprológico y Leprosería Nacional de Trillo, Centro Nacional de Lucha contra las Enfermedades Reumáticas, Centros Maternales y Pediátricos, Hospitales Rurales y Centros de Urgencias dependientes de la Dirección General de Sanidad, Centro Nacional de Rehabilitación e Instituto Español de Hematología y Hemoterapia y los Centros Nacionales de Sanidad de Majadahonda. Se integrarían, igualmente, el Centro especial de Talavera de la Reina (Toledo), Hospital General de Soria y Clinicas Infantiles de Valencia y Sevilla.

De acuerdo con lo dispuesto en esta normativa, se pueden también encomendar al citado Organismo autónomo, los Centros Comarcales 
y Subcomarcales de Sanidad, dispensarios, consultorios, centros de diagnóstico y de orientación terapéutica.

En el sector de la atención en salud pública, es preciso recordar la actividad desarrolla por las Jefaturas Provinciales de Sanidad en las que además del área de laboratorios, dispensaban atenciones preventivas en salud infantil, maternal, dermatología, oftalmología, odontología, veterinaria y el control farmacéutico, así como en ocasiones, el servicio de transfusión sanguínea.

Como ya se ha referido con anterioridad, dependiendo asimismo de la Administración Central se encuentran las instituciones asistenciales militares de tres Ejércitos, que disponen de una importante red hospitalaria e, igualmente, los Hospitales Clínicos y la Casa de Salud «Santa Cristina» y Escuela Oficial de Matronas adscritos al Ministerio de Educación, así como algunas unidades hospitalarias penitenciarias relacionadas con la Administración de Justicia.

Aunque la normativa básica deriva del Reglamento de Sanidad del año 1925, de acuerdo con lo dispuesto posteriormente en la Ley de Bases de Sanidad de 1944 y en la Ley de Régimen Local de 1955, las Corporaciones Locales -Diputaciones, Cabildos y Ayuntamientos-, regentaban un considerable número de hospitales con gran implantación provincial y local, destinados a la atención de los enfermos incluidos en los Padrones de Beneficencia o, simplemente, a ciudadanos carentes de recursos económicos.

En ocasiones, estos establecimientos asistenciales estaban vinculados con la docencia impartida por las Facultades de Medicina, bajo la denominación de hospitales provinciales y clínicos. Igualmente, las Corporaciones Provinciales se responsabilizan de la asistencia psiquiátrica en régimen de internado ya fuese con el mantenimiento de instituciones específicas propias, o bien, financiando la atención de estos pacientes en los centros de instituciones benéfico privadas.

La competencia sanitaria municipal centraba sus servicios en materia de salubridad e higiene de las localidades y, específicamente, se les atribuye a los ayuntamientos la asistencia médico-farmacéutica de las familias desvalidas. Mantienen las Casas de Socorro, los Laboratorios Municipales en los grandes Municipios, gestionan algunos centros asistenciales propios y tutelan o forman parte de los Patronatos de hospitales benéficos de ámbito local.

\section{La atención sanitaria facilitada por la Seguridad Social}

Un hecho absolutamente determinante de la organización de la asistencia sanitaria y del equipamiento institucional español, lo cons- 
tituyó la implantación y el desarrollo del Seguro Obligatorio de Enfermedad (SOE), Creado por Ley de 14 de diciembre de 1942, inició su labor asistencial en 1944 siguiendo desde entonces una tendencia expansiva, facilitando atención sanitaria a un número de afiliados y población siempre creciente, proceso que se ha realizado sobre la base de agregar diferentes colectivos.

En el año 1945 se aprueba el Plan Nacional de Instalaciones Sanitarias que no comienza a ejecutarse hasta el año 1948 y que recogía 16.114 camas de nueva construcción. A diferencia de lo ocurrido en otros países europeos, la Seguridad Social a través del INP (creado 1908), planifica y gestiona de forma paralela su propia organización sanitaria mediante centros de atención ambulatoria y hospitalaria -instituciones «abiertas» y "cerradas»-, denominando a los hospitales «Residencias Sanitarias», con una actividad más acorde con la que se realizaba en las clínicas privadas. Esta circunstancia determinó un avance sustancial en la cobertura territorial y en la mejora de la estructura y de la calidad de los centros asistenciales.

La asistencia sanitaria que da cobertura a sus afiliados se regula de forma general en la Ley de Bases de la Seguridad Social de 1966, recogidos en el Texto refundido de 1974 y el nuevo de 1994. En relación con esta materia, es obligado referirse a la aprobación del «Estatuto Jurídico del Personal Médico de la Seguridad Social», Decreto 3160/1966, de 23 de diciembre, y a la normativa sobre asistencia sanitaria de la Seguridad Social por el Decreto 2766/67, de 16 de noviembre, «Asistencia Sanitaria y Ordenación de los Servicios Médicos», que aún continúan siendo textos de referencia en la actualidad. El Estatuto del personal no sanitario al servicio de las Instituciones Sanitarias de la Seguridad Social y el del personal auxiliar sanitario titulado y auxiliar de clínica, se aprobaron en 1971 y 1973 respectivamente.

El Reglamento General para el Régimen, Gobierno y Servicios de las Instituciones Sanitarias de la Seguridad Social recogido en la Orden 7-7-1972, confirma una estructura distinta de ejercicio hospitalario, que ya se venía ejerciendo en algunos centros desde 1964, con el criterio de jerarquización de las distintas funciones en Secciones, Servicios y Departamentos, médicos y quirúrgicos, debidamente coordinados y con Servicios Generales comunes para toda la institución, con los mismos criterios de estructura.

NOTA 2. Se clasifican los centros asistenciales de la Seguridad Social en cerrados y abiertos. Son instituciones cerradas u hospitales las siguientes: Ciudades Sanitarias, Residencias Sanitarias con Servicios Regionales, Residencias Sanitarias Provinciales, Residencias Sanitarias 
Los últimos 20 años de los Centros Sanitarios en España

Comarcales. Son instituciones abiertas los Centros de Diagnóstico y tratamiento, Ambulatorios y Consultorios de Medicina General.

La bonanza económica de la que goza la Seguridad Social por aquellos años, determina que el programa de instalaciones hospitalarias del INP siga un ritmo acelerado con la construcción y puesta en funcionamiento de numerosas instituciones, en las que se desarrolla la actividad asistencial bajo el reseñado modelo: el denominado «hospital jerarquizado", distinto al que se encontraba establecido en los hospitales tradicionales de esta Entidad, los cuales también, progresivamente, se irían integrando en esta modalidad, de tal forma que el sistema asistencial que se desarrolló en la década de los años 70, significó el esfuerzo del sistema público que giró en torno a la creación de una dotación hospitalaria moderna de la que carecía nuestro país.

A finales del año 1976, se encontraban en funcionamiento 14 Ciudades Sanitarias que comprendían 49 Centros, 88 Residencias o Centros en el resto de las provincias, todo ellos con un total de 41.582 camas. En ese momento se encontraban en construcción otras 33 Residencias o Centros, con 11.303 camas.

Es preciso referir también que la Ley de Seguridad Social del año 1963 con la supresión del régimen de colaboración y la consiguiente desaparición de los Ambulatorios propiedad de las entidades colaboradoras, obligó al INP a una especial dedicación en la construcción de este tipo de instituciones, de tal forma que en el mencionado año 1976, las instituciones sanitarias abiertas o Ambulatorios totalizaban 955 establecimientos: 4 Centros de Diagnóstico y Tratamiento, 277 Ambulatorios, 281 Ambulatorios provisionales, 290 Consultorios. En esta misma fecha se encontraban en programación 2 Centros de Diagnóstico y Tratamiento, 24 Ambulatorios y 125 Consultorios.

En cuanto al personal sanitario, el total de médicos en la Seguridad Social que ascienden en el año 1976 a 47.251, representan el 81,63\% del total de los existentes en España. Los médicos especialistas alcanzaban el $64 \%$ sobre el total, con 30.199 facultativos.

En este año cerca del 84 por 100 de población se encuentra cubierta por la asistencia sanitaria de la Seguridad Social.

La financiación de la asistencia sanitaria facilitada por el INP se efectúa en el año que venimos refiriendo, en un $81,55 \%$ por cuotas de trabajadores y empresarios, transferencias el $12,52 \%$, alcanzando la aportación del Estado el 0,63\% del total de los recursos. En los pactos de la Moncloa del año 1977, se acordó que la contribución del Estado a la financiación de la Seguridad Social se incrementase hasta 
alcanzar el $20 \%$ de su presupuesto. En el año 1981, esta contribución se fija en el $10,39 \%$ de los ingresos totales del sistema, la aportación de las empresas y de los trabajados es, respectivamente, del $73,85 \%$ y del $13,14 \%$ del presupuesto de ingresos.

La asistencia en régimen de hospitalización a los beneficiarios, se hace efectiva en las instituciones sanitarias propias o en otras públicas o privadas previamente concertadas. La Seguridad Social no había orientado su política asistencial a la asistencia psiquiátrica, ni a la geriátrica, ni a la correspondiente a la patología infecciosa. El sistema de conciertos con el resto del sector público para algunas de éstas u otros tipos de atenciones, significó el mantenimiento de unas tarifas claramente insuficientes, por lo que determinaba un esfuerzo financiero complementario favorable a la Seguridad Social y, al propio tiempo, el efecto negativo de la imposibilidad del perfeccionamiento técnico y funcional de sus propias instituciones.

El sistema de conciertos con instituciones sanitarias, públicas o privadas, viene regulado por Resolución de la Secretaria de Estado para la Sanidad de 11 de abril de 1980, siguiendo la doctrina de carácter subsidiario y complementario de las prestaciones de servicios y actividades sanitarias desarrolladas por la seguridad social.

Bajo la jurisdicción del Ministerio de Trabajo, el Instituto Nacional de la Marina gestiona la asistencia sanitaria de los trabajadores del mar. Es responsable de la Sanidad Marítima de este colectivo y dispone de una red de servicios e instituciones asistenciales propios y concertados, los cuales, de forma progresiva, se están integrando actualmente en los servicios de salud de las Comunidades Autónomas con competencias en la asistencia sanitaria.

Las Mutuas Patronales son asociaciones de empresarios constituidas con la finalidad precisa y única de asumir mancomunadamente, mediante reparto, las responsabilidades asistenciales por accidentes de trabajo o enfermedades profesionales. En consecuencia, dichas Mutuas Patronales colaboran en la gestión de los Seguros Sociales de acuerdo con lo previsto en el Reglamento de colaboración en la gestión de la Seguridad Social, de 6 de julio de 1967 y diciembre de 1995.

Por Ley de 27 de junio de 1975, se crea en la Seguridad Social el régimen especial de las Fuerzas Armadas, encomendándose la gestión del Instituto Social de las Fuerzas Armadas (ISFAS), con personalidad jurídica propia de derecho público y patrimonio propio para el cumplimiento de sus fines. En la misma fecha se crea la Mutualidad General de Funcionarios Civiles del Estado (MUFACE), dependiente de la Presidencia de Gobierno y MUGEJU, para la Administración de Justicia. 


\section{Los últimos 20 años de los Centros Sanitarios en España}

Las prestaciones sanitarias son del mismo nivel que las correspondientes al régimen general de la Seguridad Social, y con las mismas modalidades de asistencia domiciliaria, ambulatoria, en régimen de internado y con carácter de urgencia. Desde 1982, sus beneficiarios pueden elegir para su asistencia, entre la que facilitan los institutos de asistencia sanitaria de la Seguridad Social, o bien, con las compañías privadas de seguros sanitarios.

Los Funcionarios de la Administración Local estaban protegidos por la Mutualidad Nacional de Previsión de Administración Local (MUNPAL), que desapareció bien entrado los años ochenta integrándose mayoritariamente su asistencia sanitaria en el régimen general.

\section{El sector privado}

El sector benéfico-particular, de titularidad religiosa o laica, disponían asimismo de un importante número de hospitales. Los laicos, representados por centros como los de la Cruz Roja, otras fundaciones de tutela municipal a los que hay que añadir un reducido número de hospitales vinculados con la Asociación de Lucha contra el Cáncer e instituciones de gran relevancia por lo que significan como referencia de la medicina española y que cuentan con una larga tradición, destacando entre ellos el Hospital de Valdecilla en Santander, El Hospital de la Santa Cruz y San Pablo en Barcelona, Basurto en Bilbao y la Fundación Jiménez Díaz en Madrid y, posteriormente, la Clínica Universitaria de Navarra.

Cada uno de estos grupos de instituciones, debe su presencia en el sector de la hospitalización a motivos diferentes. La consolidación de la Seguridad Social y la prestación directa de las atenciones a una población creciente, en buena medida en su propia red asistencial, así como el cambio en la legislación que regula la sanidad, hace que los fines fundacionales puedan verse afectados necesitando por tanto adaptarlos a las nuevas situaciones.

El sector privado constituido por entidades privadas, clínicas particulares, hospitales e instalaciones de entidades del seguro, etc., realizan acciones sanitarias preferentemente asistenciales a determinados colectivos afiliados a las mismas, sin perjuicio de atender en régimen privado y circunstancial a personas no asociadas expresamente a las mismas. La evolución del mercado del sector privado, ha venido marcada, igualmente, por la extensión del campo de aplicación de la Seguridad Social y por la aparición de una cierta demanda a consecuencia de la elevación del nivel de renta y de la presión de nuevos colectivos sobre este último sistema. 
El Seguro Libre de Enfermedad se corresponde con sociedades de distinta naturaleza jurídica, con el objeto de dar una asistencia a determinados colectivos de población integradas por personas que voluntariamente se afilian, todo ello bajo el control del Estado. Según el Reglamento de la Comisaría de Asistencia Médica y Farmacéutica, de 7 de mayo de 1957, realiza la asistencia médica voluntaria y de régimen colectivo, a través de sociedades anónimas de seguros y mutualidades de libre creación y servicios asistenciales, organizados por empresas mercantiles o industriales, organismos oficiales o corporaciones a favor de sus personas. Están reguladas últimamente por la Ley 30/1995 de ordenación y supervisión de los Seguros Privados.

Con independencia de las posibles vinculaciones a distintos sectores del sistema sanitario, los médicos con capacidad legal para el ejercicio profesional, tienen derecho al ejercicio privado sin más requisitos que el estar debidamente colegiados en el Colegio Oficial correspondiente, con los derechos y deberes que se establecen y reconocen en la Ley de Colegios Profesionales y en el reglamento vigente de la Organización Médica Colegial, así como el respeto a la normativa vigente sobre incompatibilidades.

Los establecimientos farmacéuticos, la red de oficinas de farmacia existentes al inicio de la década de los ochenta, ascendía a 15.138 centros y en el año 1992 a 18.217, que suponía 2.146 habitantes por farmacia.

\section{La evolución de la situación hospitalaria}

No es posible una comparación válida y concluyente entre los tipos de dispositivos hospitalarios del sector público dada la heterogeneidad manifiesta de los mismos, congruente en buena medida con los distintos fines atribuidos a los variados organismos señalando, no obstante, la existencia de defectos de coordinación y programación de los recursos institucionales entre los diferentes sectores señalados.

Con carácter general se refiere que en España, al igual que en resto de los países europeos así como de la mayoría de los desarrollados, los motores de la asistencia médico-sanitaria han sido la socialización y el progreso científico y tecnológico de la medicina que actuando en estrecha relación, influyen o condicionan los aspectos organizativos del sistema asistencial, su desarrollo y la ejecución de sus actividades, en la economía de la salud, la formación del personal sanitario, la manera de ejercer la medicina, en la ética profesional y en la educación sanitaria de la salud de la población. 
El hospital moderno se configura, por tanto, como el resultado de la evolución científica de la Medicina con la introducción de las nuevas tecnologías; la organización de los hospitales en departamentos y servicios de las distintas especialidades médicas y quirúrgica; la aportación de renovadas y adecuadas instalaciones técnicas e instrumentales $y$, fundamentalmente, de recursos humanos apropiados.

La introducción de la docencia y la investigación básica y clínica, han determinado asimismo nuevas y complejas funciones, cambios en la organización y estructuras, $y$, consecuentemente, el incremento del prestigio de los hospitales que han pasado a ser el lugar de referencia de la Medicina moderna.

Las áreas de consultas externas y urgencias han experimentado singulares transformaciones, en ocasiones desbordadas por la demanda, en especial estas últimas, que es el servicio de elección para atención inmediata de todas las situaciones graves o supuestamente urgentes de la comunidad.

El gran desarrollo de la especialización médico-quirúrgica y la aparición de nuevas especialidades junto con la actualización de las existentes, la exigencia y demanda de personal especializado, determinaron la necesidad de iniciar un nuevo modelo de formación. La instauración del Programa de Médicos Internos y Residentes en 1972 -del que ya existían antecedentes en algunas instituciones, la regulación recogida en la normativa específica de 1978 sobre especialidades médicas (Real Decreto 2015/1978, de 15 julio), y el Real Decreto 127/84, de 11 de enero, que ordena la formación médica especializada y la obtención del título de especialista, ofrecen una visión moderna y homologable al resto de los países de la UE.

Por otra parte, los profesionales de la Medicina han de ejercer en el hospital con criterios de cooperación y de responsabilidad en el funcionamiento del servicio con el resto del equipo profesional que lo conforma. En la Enfermería se produjeron igualmente, importantes modificaciones y avances en su formación y ejercicio profesional con el reconocimiento de sus estudios como universitarios: Diplomados en Enfermería, y con la unificación de los colegios profesionales de practicantes, enfermeras y matronas.

De forma progresiva se han ido incorporando nuevos profesionales a la actividad hospitalaria: farmacéuticos, físicos, químicos, psicólogos, biólogos, economistas y técnicos especialistas de formación profesional en diversas ramas sanitarias.

También en esta época, se inicia la investigación clínica y básica en estos centros. Es obligado referirse a los aportes que en este campo 
supuso en España el establecimiento del Fondo de Descuento Complementario de la Industria Farmacéutica y, posteriormente, en 1980, el Fondo de Investigaciones Sanitarias de la Seguridad Social (FISS), con las ayudas a la formación de investigadores, ampliación de estudios en el extranjero, financiación de proyectos y de publicaciones, congreso y reuniones científicas.

Ultimamente han tomado en parte el relevo, la Comisión Interministerial de Ciencia y Tecnología, Las Comunidades Autónomas, ciertas Fundaciones Privadas, el Plan Nacional I+D, los Programas Marco de la Unión Europea o el desarrollo de lo previsto en la Ley General de Sanidad en esta materia.

Como resumen de esta introducción, cabe señalar que el estado previo al inicio de las transferencias de los centros sanitarios asistenciales a las CC.AA, es la que se refleja en los cuadros que se exponen a continuación que ofrece la situación hospitalaria en el año 1981 y que evidencia tanto la cuantía de las instituciones como la adscripción patrimonial de los establecimientos (Tabla 1), Asimismo en la Tabla 2 se relaciona la red de instituciones sanitarias del INSALUD en el mismo año.

Tabla 1

Establecimientos hospitalarios según la finalidad y dependencia

\begin{tabular}{|l|c|c|}
\hline & Centros & Camas en funcionamiento \\
\hline Administración Central & 243 & 84.899 \\
\hline Ministerio de Sanidad y Consumo & 192 & 65.091 \\
\hline AISN & 76 & 13.051 \\
\hline INSALUD & 116 & 52.040 \\
\hline Educación y Ciencia & 11 & 8.956 \\
\hline Defensa & 37 & 10.259 \\
\hline Otros & 3 & 553 \\
\hline Administración Local & 166 & 45.398 \\
\hline Diputaciones y cabildos & 108 & 40.896 \\
\hline Municipios & 58 & 4.502 \\
\hline Beneficencia particular & 58 & 8.955 \\
\hline Iglesia & 65 & 13.820 \\
\hline Cruz Roja Española & 33 & 3.482 \\
\hline Particulares & 487 & 37.218 \\
\hline Otros & 2 & 123 \\
\hline Total & $\mathbf{1 . 0 5 4}$ & $\mathbf{1 9 3 . 8 9 5}$ \\
\hline
\end{tabular}

Fuente; INE Estadistica de Establecimientos Sanitarios 1981 


\section{Los últimos 20 años de los Centros Sanitarios en España}

Tabla 2

Equipamiento institucional del INSALUD

\begin{tabular}{|l|c|c|}
\hline & $\begin{array}{c}\text { Instituciones } \\
\text { Cerradas }\end{array}$ & Camas en Servicio \\
\hline Centros especiales & 6 & 3.950 \\
\hline Ciudades Sanitarias (integradas por 42 centros) & 14 & 20.906 \\
\hline Residencias Sanitarias & 120 & 27.537 \\
\hline $\begin{array}{l}\text { Instituciones ajenas administradas y financiadas } \\
\text { por el INSALUD }\end{array}$ & 14 & 8.890 \\
\hline Total de camas & & $\mathbf{5 6 . 4 1 7}$ \\
\hline Instituciones abiertas: & & \\
\hline Ambulatorios Generales & 473 & \\
\hline Consultorios & 495 & \\
\hline Centros de Diagnóstico y Tratamiento & 390 & \\
\hline Servicios de Urgencia & 48 & \\
\hline Dispensarios de A.T y E.P & 1.410 & \\
\hline Total abiertas propias & & \\
\hline
\end{tabular}

Fuente: INP. Información económico-funcional de las Instituciones Sanitarias. 1981.

Los hospitales de la Administración Central representan el 23 por 100 de los establecimientos y el 44 por 100 de las camas en funcionamiento. En ellos, se asistieron 1,8 millones de pacientes, de los cuales 1,4 millones (78\%), lo fueron en los del INSALUD que, a su vez, significaban el 40 por 100 del total de los ingresos y el 27,7 por 100 de las estancias producidas en total del dispositivo asistencial con régimen de internamiento.

Los de las Administración Local con un 15,7 por 100 de los establecimientos, disponían del 23,4 por 100 de las camas, en los que se produjeron el 11 por 100 de los ingresos y el 25 por 100 de las estancias, justificadas éstas por la cronicidad de los enfermos y, fundamentalmente, por depender de ella un importante número de camas psiquiátricas.

El sector privado, benéfico privado y privado, dispone de 645 establecimientos, el 61 por 100 del total, con 63.598 camas $(32,8 \%$ del total), en las que tuvieron cabida el 36,8 por 100 de los ingresos y el 33 por 100 de las estancias.

Dentro de este último sector resulta más significativo el referirse exclusivamente a los hospitales privados, particulares, que con 487 establecimientos suponen el 46,2 por 100 del total y el 19,2 por 100 de las camas en funcionamiento ( 37.218 camas), en los que ingresaron 
el 25 por 100 de los pacientes atendidos en este medio hospitalario y determinaron el 18,1 por 100 de las estancias.

NOTA 3. Los centros especiales los constituyen: Centro Nacional de Especialidades Quirúrgicas "Ramón y Cajal», Clínica «Puerta de Hierro», Centro Nacional de Especialidades Quirúrgicas «Pabellón 8» de la C.U, Centro Nacional de Silicosis, Centro Médico «Marqués de Valdecilla», Centro Nacional de Rehabilitación de Parapléjicos.

Los hospitales ajenos financiados y gestionados por el INSALUD se corresponden con: Hospital Universitario San Cecilio de Granada, Hospital Universitario de Sevilla, Hospital Universitario de Zaragoza, Gran Hospital de Estado en Madrid, Hospital del «Niño Jesús» en Madrid, Hospital Universitario «San Carlos» en Madrid, Hospital Central de la Cruz Roja Española en Madrid, Centro de Quemados de la Cruz Roja en Madrid, Hospital de la Cruz Roja en Melilla, Hospital Universitario de Salamanca, Hospital Universitario y Residencia Maternal en Valladolid, Hospital Universitario de Valencia.

\section{La creación del Ministerio de Sanidad y Seguridad Social. Promulgación de la Constitución Española de 1978}

Una de las primeras acciones emprendidas por el Gobierno tras las elecciones de 1977, fue la creación, en el mismo año, del Ministerio de Sanidad y Seguridad Social.

Teóricamente, supuso la unificación en un único Departamento de las competencias sanitarias que se encontraban dispersas en la Administración Española.

Desde el Ministerio se va a impulsar una amplia serie de actuaciones que, sin implicar una reforma general de la Sanidad, son planteamientos en gran medida nuevos. Se promulgan una serie de decretos que se adoptan con evidente precipitación, como confirmará el Tribunal Supremo, al anular dos de ellos por defectos de procedimiento en su elaboración: Normativas sobre presupuestos e indicadores de rentabilidad en los hospitales; Gobierno y Administración de los hospitales y garantías de los usuarios. Junto a ellos se elaboró el de confección del «Mapa Sanitario del Territorio Nacional».

En el año 1978 se aprueba el Real Decreto Ley-36/1978 que recoge la escisión del Instituto Nacional de Previsión dando lugar a tres entes para la gestión separada de las prestaciones económicas, la asistencia sanitaria y los servicios complementarios de la Seguridad Social: el Instituto Nacional de la Salud (INSALUD), el Instituto Nacional de 
la Seguridad (INSS) y el Instituto Nacional de Servicios Sociales (INSERSO), que junto con la Tesorería General configuran el nuevo panorama de la Seguridad Social.

El Instituto Nacional de la Salud (INSALUD), se constituye como el órgano ejecutivo de la política sanitaria de la Seguridad Social, a quien corresponde el desarrollo de las funciones y actividades precisas para garantizar las prestaciones sanitarias. Asimismo asume las funciones propias de la medicina del trabajo que se realiza por el Instituto Nacional de Medicina y Seguridad en el Trabajo, la Clínica de Enfermedades Profesionales, la Organización de los Servicios Médicos de Empresa y el Instituto Territorial de Higiene y Seguridad en el Trabajo.

Hay que señalar igualmente, que el Pleno del Congreso de los Diputados impulsó en mayo de 1980 una Resolución de Reforma Sanitaria, que al carecer de fuerza normativa, expresaba simplemente un deseo o aspiración de la Cámara en relación con la materia sanitaria. Se trata de un texto ambiguo que difícilmente podía ser base suficiente para la reforma global de la Sanidad. En cualquier caso fue el primer pronunciamiento de las Cortes sobre esta materia.

Circunstancia de absoluta transcendencia y de total repercusión en la Administración Sanitaria, lo constituyó la aprobación y promulgación de la Constitución Española del año 1978. Se define un «Estado Social» y «Democrático de Derecho», que se organiza sobre la base de un amplio reconocimiento de las Autonomías Territoriales.

El Título I determina los Derechos y los Deberes fundamentales de los ciudadanos; el Capítulo II, recoge los principios rectores de la política social y económica, en donde se encuentran encuadrados todos los mandatos constitucionales que hacen referencia a la calidad de la vida y, como componente de ésta, la Salud (Artículos 39, 40, 41, 43, $44,45,47,49,50,51$ ).

La Organización Territorial del Estado se establece en el Título VIII, así como los principios de autonomía para la gestión de sus intereses. El Capítulo III -De las Comunidades Autónomas-, especifica las competencias y las obligaciones a repartir entre la Administración Central (Artículo 149) y las Administraciones Autonómicas (Artículo 148).

\section{La evolución de los centros sanitarios durante los últimos veinte años}

El proceso de transferencias en materia sanitaria se había iniciado ya en 1977 a los que se denominaron «Entes Preautonómicos». 
Sucesivamente se perfeccionaron y completaron aquellas transferencias con la publicación de los respectivos Estatutos y la constitución efectiva de la totalidad de las Comunidades Autónomas. Así pues, una vez aprobados los Estatutos Autonómicos de las diecisiete CC.AA, recibieron las competencias de Sanidad e Higiene, esto es: salud pública y autoridad sanitaria, así como el personal, centros y servicios. En el año 1979 lo fueron a Asturias, Canarias, Cataluña, Extremadura, País Vasco; en 1980, Baleares, C. Valenciana, Galicia, Murcia; en 1981, Andalucía y Castilla-León; en 1982, Aragón, Cantabria, Castilla La Mancha; en 1984, Madrid y la Rioja y, en 1985, Navarra.

La progresiva integración a las distintas redes asistenciales se inició con la transferencia a las CC.AA que lo desearon, de los hospitales y centros asistenciales vinculados al Ministerio de Sanidad a través de la Administración Institucional de la Sanidad Nacional (AISN). En 1980, Cataluña y el País Vasco; en 1982, Aragón, Asturias, Baleares, Castilla La Mancha, C. Valenciana, Extremadura y Murcia; en 1985, Andalucía, Castilla León, Galicia y Madrid. El resto de hospitales de este organismo que no fueron asumidos por las CC.AA, se integraron en la red asistencial del INSALUD de acuerdo con lo previsto en el artículo 93 de la Ley de Presupuestos Generales para el año 1985. Además, la disposición adicional vigésima tercera de la citada Ley, determina la integración de los Hospitales Clínicos Universitarios, dependientes del Ministerio de Educación y Ciencia, en la red hospitalaria de la Seguridad Social. (Ambas integraciones se materializaron mediante lo dispuesto en el R.D. 187/1987, de 23 de enero y en la Orden de 13 septiembre de 1985, y por otras normativas específicas).

Por otra parte, las CC.AA uniprovinciales tuvieron que hacerse cargo de los centros y servicios vinculados a sus respectivas Diputaciones Provinciales. En las restantes Comunidades, pluriprovinciales, la integración de los hospitales -generales o psiquiátricos- en sus propios servicios de salud, han seguido un camino distinto de acuerdo con lo que establecen sus estatutos de autonomía y la evolución de las redes de asistencia sanitaria.

En el año 1981 se inicia la transferencia del INSALUD a la Comunidad Autónoma de Cataluña. En la actualidad, sólo siete de ellas han recibido las transferencias de la asistencia sanitaria de la Seguridad Social, las que accedieron a la autonomía por la vía del Artículo 151 de la Constitución y, con carácter excepcional, las asimiladas, de acuerdo con una Ley orgánica específica anexa a su Estatuto de Autonomía correspondiente, por las Comunidades Autónomas de Valencia y Canarias, que accedieron a la autonomía por la vía del Artículo 143, 
debiendo las restantes que accedieron a la autonomía por esta vía, esperar a que se cumplan las previsiones constitucionales. Así, en 1984 se transfiere a Andalucía; en 1987, a la C. Valenciana y País Vasco; en 1990 a Galicia y Navarra y en 1994, a Canarias.

El INSALUD, por tanto, se responsabiliza de la gestión de los servicios asistenciales de aquellas CC.AA, a quienes no se les ha transferido estas competencias, que se extiende en la actualidad a algo menos del 40 por 100 de la población.

Las Comunidades Autónomas comienzan a ejercer en este período junto a las competencias de «ejecución» sobre la Sanidad, las de «desarrollo legislativo» de esta materia a través de la elaboración de una normativa propia legal y reglamentaria. Crean y estructuran los Servicios de Salud de las Comunidades Autónomas. Las leyes de constitución de los respectivos servicios de salud se han producido entre los años 1983 y 1994, quedando algunas de ellas pendientes de aprobación.

Como ejemplo de la evolución que han seguido en esta materia las distintas Comunidades Autónomas, cabe referirse a lo acontecido en Cataluña que en 1983 creó el Instituto Catalán de la Salud prestando servicios directamente en los hospitales propios, que son los hospitales que se transfirieron. Además asumió dos funciones añadidas: la planificación sanitaria y la financiación del sector concertado. En 1990, se promulgó la Ley de Ordenación Sanitaria de Cataluña.

Desde 1981, existe en esta Comunidad un sistema mixto de previsión de los servicios de asistencia sanitaria, con una separación previa de la función de financiación de la previsión. Hay que señalar como hecho diferencial en Cataluña, que el 50 por ciento de la oferta sanitaria no era pública. En este contesto, la Red Hospitalaria de Utilización Pública de Cataluña (XHUP), se encuentra integrada por hospitales de titularidad múltiple: Instituto Catalán de la Salud, Unión Catalana de Hospitales, Consorcio Hospitalario de Cataluña, centros del IMAS (Hospitales Municipales de Barcelona) además del Hospital de la Santa Cruz y San Pablo y el Hospital Clínico de Barcelona. Incluye, además, a todos aquellos centros que tradicionalmente habían prestado asistencia concertada, fuesen públicos o privados.

NOTA 4. En esta materia de ordenación de la asistencia especializada y órganos de dirección de hospitales hay que recordar el Decreto 105/86 de la Comunidad Autónoma de Andalucia; el 148/91 de la de Galicia; el 31/87 de la de Madrid; el 69/86 de Murcia; el 443/91 de Navarra, el 194/96 del País Vasco, el 27/92 de la Rioja y el 186/96 de la Comunidad Valenciana. 
La potenciación de la atención primaria prestada por la Seguridad Social se hace a través de distintas normativas promulgadas en el año 1992 y que culminan en el ámbito del INSALUD con el Real Decreto 137/1984, de 11 de enero, sobre estructuras básicas de salud, que viene a crear las demarcaciones territoriales denominadas Zonas de Salud, en cuyo marco operan los Centros de Salud. La cobertura por los Equipos de Atención Primaria alcanzaba en toda España en el año 1994, al 61 por 100 de la población, con notables diferencias entre las Comunidades Autónomas: del $87 \%$ en Castilla-La Mancha y el $42 \%$ en Cataluña.

Una nueva normativa en materia de hospitales, que se proyecta también sobre los centros del INSALUD, lo constituye la Orden de 28 de febrero de 1985, sobre los órganos de dirección de los hospitales y dotación de su personal, regulando la provisión de los cargos y puesto correspondiente, que fue declarada nula por sentencia del Tribunal Supremo en 1996, pero la materia objeto de regulación se recoge en el nuevo Reglamento de Estructura, Ordenación y Funcionamiento de los Hospitales gestionados por el INSALUD, aprobado por R.D. 521/1987 de 15 de abril, que viene a sustituir el Reglamento de Instituciones Sanitarias del año 1972.

En el panorama sanitario de los años que estamos exponiendo, es preciso referirse a las repercusiones que ha tenido en el ejercicio del personal sanitario, el desarrollo de la Ley de incompatibilidades, y la aprobación de la Ley General para la Defensa de los Consumidores. Aunque, en principio, podría sugerirse que esta última disposición tuviese escasa proyección sobre la asistencia sanitaria, lo cierto es que, en ocasiones su contenido es utilizado como referencia y apoyo legal de numerosas sentencias dictadas por los jueces, junto con el contenido de los artículos 9 y 10 de la Ley General de Sanidad -derechos y obligaciones de los usuarios- por reclamaciones de mala práctica y negligencias médicas.

\section{La aprobación de la Ley General de Sanidad}

A finales de 1982, después de la elecciones generales del 28 de octubre, se produce el relevo en la Sanidad por el Partido Socialista. El período 1982-1986, culminará con la aprobación de la Ley General de Sanidad.

La Ley 14/1986, de 25 de abril, General de Sanidad -se trata de una ley básica o Ley de Bases- viene a responder a una necesidad que se había dejado sentir desde hacía muchos años. Era además 
precisa, debido a la evolución social y sanitaria, la transformación institucional y legislativa experimentada en nuestro país para articular la acción pública en el marco de un Estado descentralizado, cuestión en la que coincidían todos los Grupos Parlamentarios, y que estaba incluida en el programa electoral del PSOE. Se abandona la idea de un Servicio Nacional de Salud único para toda la nación, que se juzga incompatible con la estructura autonómica del Estado y en su lugar aparece la referencia al Sistema Nacional de Salud.

Señalan los analistas del contenido de la Ley, a nuestro juicio acertadamente, que en realidad difícilmente se puede decir que la LGS opere en si misma una reforma global de la Sanidad, pero viene a actuar, eso sí, en línea con una serie de planteamientos y reformas que ya se habían ido penetrando con anterioridad en nuestra Administración Sanitaria. Contiene a su vez, un amplio programa de acción para el futuro, pero en todo caso, en una clara línea de continuidad con lo existente.

El principal logro en su haber consiste, en realidad, en la creación de un marco general -organizativo y competencial- necesario para la acción pública sanitaria del Estado descentralizado, partiendo para ello de una concepción global de la Sanidad y tratando de compatibilizar la autonomía con la existencia de un único sistema sanitario que atienda los valores de igualdad y solidaridad, creando un cuadro de referencia tanto para el desarrollo de una acción sanitaria por las Comunidades Autónomas como para el ejercicio de las competencias estatales.

La Ley General de Sanidad, ordena en su Título III el sistema sanitario público de tal forma que todas las estructuras y servicios públicos al servicio de la salud se integrarán en el Sistema Nacional de Salud, que se entiende con el conjunto de los Servicios de Salud de la Administración del Estado y de los Servicios de Salud de las Comunidades Autónomas, en los términos establecidos en la Ley.

Por tanto, en cada CC.AA se constituirá un Servicio de Salud integrados por todos los centros, servicios y establecimientos de la propia Comunidad, Diputaciones y Ayuntamientos y cualesquiera otras administraciones territoriales intracomunitarias, que estará gestionado bajo la responsabilidad de la respectiva Comunidad Autónoma.

El Capítulo Tercero de la Ley -De las Areas de Salud- establece entre los artículos 56 a 69, la organización territorial de estas Areas y de las Zonas básicas de Salud, como marco territorial de la atención primaria, en donde se desarrollan las actividades sanitarias los Centros de Salud. Los artículos 66 y 67, señalan que los hospitales generales 
del sector privado que lo soliciten serán vinculados al Sistema Nacional de Salud, mediante convenios singulares.

Como consecuencia de lo dispuesto, se ha ido pasando de un sistema asistencial basado en tres niveles -primaria, especializada extrahospitalaria y hospitalaria- a otro de dos niveles: atención primaria y atención especializada, aunque existen una serie de particularidades.

En materia de Financiación Sanitaria, la Ley General de Sanidad en su artículo 82, establece los criterios de asignación de recursos a las Comunidades que tuvieran competencias para asumir las funciones de la asistencia sanitaria de la Seguridad Social, la financiación de estos servicios transferidos se realizará siguiendo el criterio de población transferida. No obstante la propia Ley contempla la aplicación de un período transitorio de 10 años de acercamiento del criterio de coste de los servicios, que es el que debe aplicarse en el momento en que se realiza la transferencia, hasta el criterio de población protegida al cual se debe converger.

Con la aprobación del Real Decreto de 1088/89 con la incorporación de colectivos no cubiertos por el Sistema de Seguridad Social y por tanto sin cobertura sanitaria, se incrementan las dificultades para la determinación de población protegida, por lo que el criterio aplicados ha sido de coste efectivo de los servicios transferidos hasta alcanzar el criterio poblacional y de acercamiento a población protegida según los distintos censos.

El Modelo de financiación sanitaria aplicado durante el período 19941997, fue aprobado por el Pleno de Política Fiscal y Financiara en su reunión celebrada el 21 de septiembre de 1994, y el aprobado por el referido Consejo, en noviembre de 1997 para los años 1998-2001. Las características fundamentales de este último modelo contemplan la aportación de recursos adicionales al Acuerdo de Financiación del año 1998, la garantía del equilibrio financiero vinculado a la tasa de variación del PIB nominal, la articulación del Modelo de Financiación 1998-2001 en Fondos, Criterios de distribución de los recursos y Programas de Racionalización del Gasto.

Hay que referir en este apartado la aportación del Estado a la financiación de la asistencia sanitaria pública de tal forma, que en el año 1997 alcanzo el 70,20\% de su importe, aumentándose el porcentaje hasta llegar al 91,9\% en 1997 y en el pasado año de 1999, prácticamente su totalidad lo fue con cargo a los presupuestos generales.

\section{Análisis de la evolución del equipamiento y de la actividad hospitalaria}

Existen evidentes diferencias en el equipamiento hospitalario de las distintas Comunidades Autónomas, tanto en su cuantía global como 
en la distribución entre la dependencia pública y privada de los centros y camas. Se ha producido una disminución muy significativa en el número de centros hospitalarios, pasando respectivamente de 1.287 en 1972 a 799 en 1997, pero ha mejorado sensiblemente la accesibilidad a este tipo de asistencia debido a la construcción de nuevos hospitales en las áreas metropolitanas de las grandes ciudades y los de ámbito comarcal.

Las circunstancias que justifican esta disminución del número de hospitales han sido el cierre o desafectación del Catálogo de un gran número de establecimientos de carácter asilar destinados a la atención social de personas de la tercera edad, al cierre de un importante número establecimientos privados de escasa entidad asistencial y al agrupamiento de éstos establecimientos en complejos hospitalarios cuando, con anterioridad, se contabilizaban de forma independiente. Obviamente, se ha incremento el tamaño medio de los centros hospitalarios.

Aunque no de una forma tan manifiesta, ha disminuido asimismo el número de camas instaladas, de 177.385 en 1972, a 202.969 en el año 1981 a 166.276 en 1997, de acuerdo con lo refleja el último Catálogo Nacional de Hospitales.

La cuantía de las camas creadas en los nuevos hospitales, no ha compensado a las de los establecimientos que se desafectaron a las que se suman las reducidas en los grandes centros hospitalarios con el objeto de agilizar su función, modernizar sus instalaciones, mejorar la actividad asistencial o dar cabida a nuevas áreas funcionales o de asistencia ambulatoria.

La disponibilidad de camas por 1.000 habitantes ha evolucionado desde 5,3 camas en $1972 ; 5,13$ en $1981 ; 4,56$ en $1985 ; 4,26$, en 1990 y 3,94 en 1995. Este descenso no ha sido tan acusado en las camas de agudos, que de 3,40 camas por 1.000 habitante en 1992 se pasa a 3,09 en 1994, lo que significa que se han mantenido relativamente estable.

En la actividad desarrollada por los hospitales ha aumentado, discretamente, el número de altas que en los últimos años alcanzan más de 4 millones; se ha mantenido el número de estancias entre $45 \mathrm{y}$ 50 millones y se han incrementado de forma espectacular el número de consultas externas. La fuerte presión de la atención de urgencias, determina que más de la mitad de los ingresos hospitalarios se produzcan desde este área asistencial, representando un 13, 38 por 100 de los pacientes que son atendidos con este carácter.

Se ha incrementado, igualmente, el personal que trabaja en los hospitales. En el año 1981 lo hacían 278.582 personas que suponía 
1,44 personas cama, correspondiendo al sector público el 72,5 del total. En año 1995, su cuantía ascendía a 370.244 de las cuales el 77,8 por 100 correspondía al sector público, 2,22 persona cama. En este período de tiempo el personal de los hospitales se incrementó en un 42,6 por 100. (Tabla 4).

En los indicadores del funcionamiento hospitalario global, se manifiesta en un progresivo incremento de la frecuentación hospitalaria y de tasa de ocupación, el descenso de la estancia media y, consecuentemente, un incremento de la rotación enfermo/cama.

En los aspectos cualitativos de la función hospitalaria, como ya se ha señalado con anterioridad, en las últimas décadas se ha producido una decisiva transformación en la organización y funcionamiento de la asistencia médica con la mejora de la calidad. El hospital ha adquirido una complejidad creciente de servicios, instalaciones y de las funciones que desarrolla, con el aumento de personal cualificado, así como una carrera de costes crecientes. Se han implantado nuevos organigramas con la diversificación de áreas y zonas funcionales, nuevas tecnologías que evitan los ingresos y favorecen la atención externa así como, en ocasiones, la introducción de guías o protocolos en proceso asistencial.

La cobertura sanitaria pública en 1982 incluía al 84,5 por 100 de población, en 1991 este porcentaje era superior al 98 por 100 . La última Encuesta Nacional de Salud de España que corresponde al año 1997, el 94,8 por 100 de los encuestados son titulares o beneficiarios de la Seguridad Social; el 2,3 por 100 a Mutualidades del Estado acogidas a la Seguridad Social; Mutualidades del Estado acogidas a un seguro privado, el $2,3 \%$ acogidas a un seguro privado. Se produce un claro solapamiento en la cobertura de la asistencia sanitaria por cuanto que un 8,9 por 100 de las contestaciones recogidas manifiestan disponer de un seguro médico privado, concertado individualmente y el 2 por 100 de un Seguro médico concertado por su empresa.

En cuanto a los centros benéfico privado o privados, se puede observar la marcada especialización de los centros de la Iglesia en la asistencia psiquiátrica, representando el $34,4 \%$ de las camas de esta especialidad, la reducida capacidad media, la importancia de los centros quirúrgicos de pequeñas dimensiones y la desigualdad en el reparto de las clínicas privadas en el territorio nacional.

Los centros hospitalarios no públicos ascienden en el año 1995 a 458 los cuales contabilizan un total de 50.112 camas, en los que se produjeron 1,3 millones de ingresos, 14 millones de estancias, 7,5 mi- 


\section{Los últimos 20 años de los Centros Sanitarios en España}

llones de consultas externas y 3,7 millones de urgencias. Los exclusivamente privados, que representan el 19,5 por 100 de las camas totales, asistieron al $21,5 \%$ de los ingresos, que determinaron el $17,51 \%$ de las estancias, el $14,8 \%$ de las consultas externas y el $15,63 \%$ de las urgencias.

Tabla 3

Número de hospitales por dependencia patrimonial

\begin{tabular}{|l|c|c|}
\hline & Hospitales & Camas instaladas \\
\hline Seguridad Social & 130 & 54.949 \\
\hline Administración central & 3 & 636 \\
\hline Defensa & 17 & 5.766 \\
\hline Comunidad Autónoma & 92 & 22.019 \\
\hline Diputación o Cabildo & 47 & $\mathbf{8 . 4 5 5}$ \\
\hline Municipio & 18 & 2.391 \\
\hline Entidades públicas & 28 & 19.648 \\
\hline Mutualidades Acc., Trabajo & 24 & 1.846 \\
\hline Privado benéfico (C. Roja) & 17 & 2.071 \\
\hline Privado benéfico (Iglesia) & 64 & 12.286 \\
\hline Otros priv. benéficos & 63 & 8.037 \\
\hline Privado no benéfico & 296 & 28.172 \\
\hline Total & 799 & 166.276 \\
\hline
\end{tabular}

Fuente: Catálogo Nacional de Hospitales a 3-12-97 M.S y C.

En las Tablas 5 y 6, se reflejan la evolución del equipamiento y movimiento hospitalario por Comunidades Autónomas y en la Tabla 7, indicadores del grado de utilización.

En el último Catálogo de Hospitales correspondiente a finales del año 1997, el número de hospitales ascendía a 799, con la distribución de centros y camas que se manifiesta en la Tabla 3.

En el sector extrahospitalario se han instaurados un gran número de servicios o centros que además de los Centros de Salud y Consultorios ya referidos, se relacionan, entre otros, las Salas de fisioterapia, Unidades de psicoprofilaxis obstétrica, Unidades de Salud Mental, Unidades de Medicina laboral, centros de orientación familiar, Centros de Día, Servicios especiales de urgencia, centros de atención continuada, servicios normales de urgencia, etc. 


\section{Víctor Conde Rodelgo}

Tabla 4

Evolución de los indicadores sanitarios

\begin{tabular}{|l|c|c|c|c|}
\hline & $\mathbf{1 9 8 1}$ & $\mathbf{1 9 8 5}$ & $\mathbf{1 9 9 0}$ & $\mathbf{1 9 9 5}$ \\
\hline Camas funcionamiento por 1000/Hab. & 5,13 & 4,56 & 4,26 & 3,94 \\
\hline Altas por 1000 habitantes & 91,69 & 93,17 & 97,34 & 108,82 \\
\hline Tasa de Ocupación & $71,46 \%$ & $75,22 \%$ & $76,19 \%$ & $79,05 \%$ \\
\hline Estancia Media & 14,59 días & 13,42 días & 12,17 días & 10,46 días \\
\hline Personal/Cama & 1,44 & 1,65 & 2,10 & 2,22 \\
\hline
\end{tabular}

Tabla 5

Distribución de hospitales y camas por Comunidades Autónomas

\begin{tabular}{|c|c|c|c|c|c|c|c|c|c|c|}
\hline \multirow[t]{2}{*}{$\begin{array}{l}\text { COMUNIDADES } \\
\text { AUTÓN. }\end{array}$} & \multicolumn{5}{|c|}{ Número de Hospitales } & \multicolumn{5}{|c|}{ Camas en funcionamiento } \\
\hline & 1981 & 1985 & 1990 & 1995 & 1997 & 1981 & 1985 & 1990 & 1995 & 1997 \\
\hline Andalucía & 149 & 120 & 95 & 84 & 88 & 28898 & 26356 & 23244 & 21872 & 23761 \\
\hline Aragón & 41 & 35 & 27 & 27 & 27 & 7790 & 6653 & 6147 & 5431 & 6089 \\
\hline Asturias (Principado) & 31 & 27 & 27 & 24 & 23 & 6514 & 5629 & 4726 & 4052 & 4729 \\
\hline Baleares (Islas) & 22 & 21 & 20 & 19 & 24 & 3865 & 3490 & 3223 & 3110 & 3668 \\
\hline Canarias & 51 & 52 & 50 & 49 & 50 & 7566 & 8219 & 7947 & 7658 & 8181 \\
\hline Cantabria & 15 & 13 & 10 & 11 & 11 & 3180 & 2509 & 2204 & 2231 & 2787 \\
\hline Castilla-La Mancha & 44 & 36 & 31 & 31 & 30 & 6817 & 5779 & 5515 & 5178 & 5586 \\
\hline Castilla-León & 78 & 68 & 61 & 53 & 56 & 16020 & 14475 & 13727 & 11756 & 10501 \\
\hline Cataluña & 193 & 175 & 177 & 176 & 180 & 31195 & 29262 & 30560 & 29897 & 31781 \\
\hline Comu. Valenciana & 75 & 58 & 54 & 56 & 61 & 15067 & 12750 & 11921 & 11712 & 12991 \\
\hline Extremadura & 24 & 22 & 18 & 17 & 18 & 4268 & 4436 & 4299 & 4021 & 4350 \\
\hline Galicia & 96 & 78 & 67 & 63 & 59 & 11553 & 10423 & 11064 & 10719 & 11217 \\
\hline Madrid & 106 & 94 & 81 & 75 & 74 & 27499 & 24592 & 22579 & 20748 & 23231 \\
\hline Murcia (Región de) & 26 & 27 & 23 & 23 & 25 & 4133 & 4182 & 3601 & 3459 & 4216 \\
\hline Navarra & 15 & 15 & 13 & 14 & 14 & 3782 & 3367 & 2930 & 2609 & 2694 \\
\hline País Vasco & 75 & 61 & 57 & 51 & 51 & 12518 & 10784 & 10155 & 8759 & 8923 \\
\hline La Rioja & 8 & 7 & 5 & 5 & 4 & 2068 & 1699 & 1301 & 948 & 1036 \\
\hline Ceuta & 3 & 2 & 2 & 2 & 2 & 619 & 486 & 352 & 233 & 263 \\
\hline Melilla & 2 & 2 & 2 & 2 & 2 & 543 & 319 & 402 & 251 & 272 \\
\hline TOTALES & 1054 & 913 & 820 & 782 & 799 & 193895 & 175410 & 165897 & 154644 & 166.276 \\
\hline
\end{tabular}




\section{Los últimos 20 años de los Centros Sanitarios en España}

Tabla 6

Altas y estancias por Comunidades Atónomas

\begin{tabular}{|l|c|c|c|c|c|c|c|c|}
\hline $\begin{array}{c}\text { COMUNIDADES } \\
\text { AUTÓN. }\end{array}$ & \multicolumn{7}{|c|}{ N $^{\mathbf{0}}$ de Altas Totales } & \multicolumn{3}{c|}{ Número de Estancias Totales } \\
\hline & $\mathbf{1 9 8 1}$ & $\mathbf{1 9 8 5}$ & $\mathbf{1 9 9 0}$ & $\mathbf{1 9 9 5}$ & $\mathbf{1 9 8 1}$ & $\mathbf{1 9 8 5}$ & $\mathbf{1 9 9 0}$ & $\mathbf{1 9 9 5}$ \\
\hline Andalucía & 583068 & 614338 & 632559 & 697286 & 7.410 .775 & 6.912 .022 & 6.354 .430 & 6.091 .386 \\
\hline Aragón & 109354 & 115590 & 126882 & 136739 & 1.813 .690 & 1.778 .286 & 1.614 .088 & 1.548 .856 \\
\hline Asturias (Principado) & 106529 & 105131 & 104625 & 115910 & 1.638373 & 1.455 .494 & 1.277 .693 & 1.213 .374 \\
\hline Baleares (Islas) & 77190 & 85419 & 91567 & 110510 & 935.390 & 857.184 & 844.437 & 886.722 \\
\hline Canarias & 116773 & 125788 & 132840 & 159935 & 2.120 .055 & 2.268 .968 & 2.310 .386 & 2.286 .507 \\
\hline Cantabria & 50152 & 45554 & 48940 & 50772 & 745.521 & 662.478 & 653.881 & 679.926 \\
\hline Castilla-La Mancha & 118684 & 123794 & 129309 & 151416 & 1.750 .012 & 1.599 .214 & 1.490 .605 & 1.513 .440 \\
\hline Castilla-León & 175694 & 238401 & 232981 & 255255 & 4.390 .358 & 4.163 .589 & 3.776 .020 & 3.406 .814 \\
\hline Cataluña & 596426 & 619881 & 701291 & 789264 & 8.709 .012 & 8.638 .463 & 9.008 .098 & 9.071 .322 \\
\hline Comu. Valenciana & 307655 & 319024 & 337782 & 396503 & 3.763 .870 & 3.449 .368 & 3.263 .837 & 3.198 .015 \\
\hline Extremadura & 80057 & 92942 & 92111 & 100503 & 1.083 .977 & 11.877 .437 & 1.117 .671 & 1.188 .491 \\
\hline Galicia & 213675 & 211950 & 237673 & 282109 & 3.014 .771 & 2.833 .542 & 3.129 .319 & 3.086 .01 \\
\hline Madrid & 451030 & 463553 & 495811 & 557018 & 7.243 .077 & 6691178 & 6.213 .928 & 5.775 .456 \\
\hline Murcia (Región de) & 91814 & 95503 & 107675 & 110170 & 1.087 .003 & 1.066 .416 & 911.096 & 998.458 \\
\hline Navarra & 58140 & 63786 & 63763 & 69929 & 991.902 & 973.905 & 848.706 & 750.133 \\
\hline País Vasco & 219836 & 222615 & 214505 & 242295 & 3.232 .312 & 3.039 .191 & 2.820 .883 & 2.513 .033 \\
\hline La Rioja & 28263 & 27316 & 27156 & 24473 & 496.438 & 418.989 & 372.999 & 300.226 \\
\hline Ceuta & 7633 & 9401 & 8880 & 9200 & 82.352 & 90.942 & 67.223 & 58.859 \\
\hline Melilla & 6033 & 7496 & 5749 & 7392 & 63.880 & 74.137 & 57.698 & \\
\hline TOTALES & $\mathbf{3 . 4 4 5 . 7 9 1}$ & $\mathbf{3 . 5 8 7 . 5 8 5}$ & 3.792 .099 & $\mathbf{4 . 2 6 6 . 6 7 9}$ & $\mathbf{5 0 . 5 7 3 . 3 6 8}$ & $\mathbf{4 8 . 1 6 0 . 8 0 3}$ & 46.132 .998 & $\mathbf{4 4 . 6 2 1 . 2 0 1}$ \\
\hline & & & & & & & & \\
\hline
\end{tabular}

\section{La situación actual en la gestión de los hospitales}

La creciente competitividad mundial, condicionada por las diferencias en los costes y estructuras laborales y en la productividad, la crisis económica y socio-laboral acaecida en los años setenta y, en nuestro entorno, las exigencias en el proceso de convergencia entre los países de la UE acordado en Maesttrich, son algunas de las circunstancias que han determinando profundos cambios en los esquemas económicos imperantes en los países industrializados. 
Tabla7

Altas, consultas externas y urgencias por 1000 habitantes

\begin{tabular}{|c|c|c|c|c|c|c|c|c|c|c|c|c|}
\hline \multirow[t]{2}{*}{$\begin{array}{l}\text { COMUNIDADES } \\
\text { AUTÓNOMAS }\end{array}$} & \multicolumn{4}{|c|}{$\begin{array}{c}\text { Frecuentación } \\
\text { Hospitalaria/1000 }\end{array}$} & \multicolumn{4}{|c|}{ Consultas Externas/1000 Hab. } & \multicolumn{4}{|c|}{ Número de Urgencias/1000 hab } \\
\hline & 1981 & 1985 & 1990 & 1995 & 1981 & 1985 & 1990 & 1995 & 1981 & 1985 & 1990 & 1995 \\
\hline Andalucia & 94,71 & 92,21 & 91,41 & 98,26 & 454,98 & 439,66 & 558,09 & 764,34 & 139,55 & 250,98 & 353,10 & 424,03 \\
\hline Aragón & 94,07 & 95,61 & 104,64 & 115,66 & 751,90 & 706,87 & 1400,90 & 1092,05 & 89,36 & 252,08 & 337,20 & 397,10 \\
\hline Asturias (Principado) & 93,81 & 92,61 & 92,92 & 107,67 & 485,16 & 464,54 & 733,37 & 848,12 & 50,15 & 175,02 & 238,80 & 318,66 \\
\hline Baleares (Islas) & 117,25 & 127,50 & 134,19 & 152,31 & 556,66 & 576,49 & 779,99 & 859,88 & 106,39 & 377,99 & 444,52 & 539,04 \\
\hline Canarias & 78,12 & 88,30 & 89,46 & 103,23 & 387,88 & 487,83 & 567,47 & 711,95 & 75,00 & 235,45 & 309,62 & 353,77 \\
\hline Cantabria & 99,21 & 87,23 & 92,83 & 96,41 & 594,23 & 440,08 & 647,96 & 713,94 & 39,25 & 263,93 & 348,66 & 445,200 \\
\hline Castilla - La Mancha & 77,15 & 74,49 & 75,46 & 89,76 & 366,74 & 334,74 & 621,94 & 929,75 & 48,89 & 126,40 & 203,68 & 319,25 \\
\hline Castilla - León & 84,26 & 91,97 & 88,72 & 101,46 & 544,82 & 494,45 & 850,16 & 887,82 & 101,01 & 166,29 & 257,04 & 310,10 \\
\hline Cataluña & 96,21 & 102,92 & 116,73 & 130,08 & 874,88 & 784,07 & 948,01 & 1136,37 & 183,34 & 358,85 & 481,65 & 554,32 \\
\hline Comun. Valenciana & 83,00 & 85,00 & 89,20 & 101,58 & 463,41 & 461,01 & 431,98 & 624,98 & 112,68 & 219,37 & 309,66 & 403,98 \\
\hline Extremadura & 82,31 & 86,22 & 81,65 & 93,72 & 249,92 & 293,26 & 509,85 & 538,70 & 42,78 & 104,18 & 221,68 & 318,70 \\
\hline Galicia & 76,89 & 74,30 & 84,75 & 103,46 & 342,73 & 310,57 & 547,76 & 787,08 & 52,73 & 153,34 & 227,57 & 327,47 \\
\hline Madrid & 91,77 & 95,52 & 101,64 & 111,21 & 646,53 & 797,97 & 783,49 & 942,56 & 113,87 & 245,87 & 358,89 & 427,96 \\
\hline Murcia (Región de) & 100,08 & 95,99 & 104,88 & 102,24 & 431,18 & 311,70 & 692,62 & 832,89 & 106,70 & 220,00 & 356,22 & 433,95 \\
\hline Navarra & 117,18 & 123,01 & 122,32 & 133,12 & 599,97 & 608,45 & 782,12 & 905,62 & 67,22 & 343,78 & 389,23 & 424,22 \\
\hline País Vasco & 97,04 & 102,85 & 100,74 & 116,69 & 409,09 & 512,85 & 617,79 & 807,28 & 101,26 & 233,05 & 293,37 & 370,97 \\
\hline La Rioja & 117,10 & 104,76 & 104,08 & 93,81 & 806,27 & 353,46 & 1029,65 & 1115,20 & 148,28 & 173,29 & 272,42 & 334,15 \\
\hline Ceuta y Melilla & 113,37 & 140,17 & 116,80 & 126,03 & 636,42 & 867,02 & 998,95 & 951,19 & 56,57 & 109,02 & 335,87 & 562,87 \\
\hline
\end{tabular}

Fuente: I.N.E.

INE. Estadística de Establecimientos Sanitarios con Régimen de Internado

Catálogo Nacional de Hospitales Diciembre1997. Camas instaladas 
En las últimas décadas, están obligando a una mayor disciplina presupuestaría y a una regulación y reducción del déficit público que, lógicamente, comprometen la cobertura de las crecientes demandas de los sistemas de atención social e incluso, su mantenimiento en los niveles actuales.

Con carácter general existe un consenso generalizado sobre la necesidad de continuar manteniendo el Estado de Bienestar aunque, últimamente, en algunos medios se empieza a hablar de pasar a la Sociedad del Bienestar, concepto que implicaría una mayor participación y preocupación de la Sociedad en su permanencia.

Se reconoce que el Estado de Bienestar es una conquista del sistema democrático y que, en consecuencia, debe mantenerse, partiendo de la base de que el objetivo último del mismo consiste en colaborar en la consecución de la Economía del Bienestar, como un medio de alcanzar cotas más elevadas de bienestar social y no como un fin en si mismo.

El análisis de la evolución del gasto en sanidad en las ultimas décadas pone de manifiesto que a partir de la crisis del año 1973-74, determinada por la subida de los precios del petróleo y de las materias primas así como otros problemas estructurales de la actividad económica mundial, se produjo una reducción de la tasa de crecimiento del PIB. La totalidad de los países desarrollados se enfrentaron a una insuficiencia de recursos para atender el gasto público. La inercia de demandas de atenciones sociales crecientes, y las sanitarias en concreto, obligaron a adoptar medidas de variada naturaleza para la contención del gasto social en general y del sanitario, en particular.

Entre las medidas adoptadas para la contención del gasto, cabe destacar, el estudio y valoración de los sistemas sanitarios establecidos en los distintos países y la aportación de propuestas de modificaciones o de actuaciones restrictivas concretas.

Es preciso referirse a la inquietud suscitada en este sector a nivel internacional: Reforma Blüm y Seehofer (1992), en Alemania; Informe Abril (1991) en España; Informe Dunning y Plan Dekker en Holanda (1988-1991); Plan Griffiths y el Libro Blanco de la Reforma Working for Patients en el Reino Unido; Informe Dogmar en Suecia; además habría que citar las reformas adoptadas en Canadá y los intentos fallidos en los Estados Unidos (Plan Clinton).

En España, la Comisión Evaluadora del Sistema Nacional de Salud, «Comisión Abril», aprobó un conjunto de 64 recomendaciones orientadas a la organización y gestión del Sistema Nacional de Salud y para la reforma de la asistencia sanitaria pública.

De entre éstas cabe destacar las siguientes referidas a la gestión de la asistencia: la descentralización, el separar la financiación de los 
servicios de la previsión, flexibilidad de personal, reforzar los sistemas de información, la evaluación de los avances tecnológicos, mayor calidad de los servicios, contratación externa de servicios, previsión pública y privada, estrategias de gestión y la distinción entre la atención sanitaria y la social.

La "Comisión Abril» se ofreció como un especial lugar de encuentro para el análisis de la situación de la sanidad española y para emitir importantes propuestas, que si bien no fueron admitidas formalmente por la Administración, han supuesto un buen lugar de referencia para las iniciativas que se han adoptado posteriormente.

El grado de implantación de las reformas, y muy particularmente, las que afectan a la organización y gestión de los proveedores sanitarios es muy variable entre los países, dependiendo de la capacidad de conseguir recursos políticos y sociales para afrontar los cambios y superar la rigidez estructural de la que se parte históricamente.

En la gestión sanitaria actual, se están introduciendo nuevos conceptos y modelos a través de mecanismos de descentralización y competitividad con la introducción del denominado «mercado interno», dotándolo de un sentido empresarial y diferenciando la financiación, la gestión de la demanda y la compra de servicios, de la previsión o suministradores de los mismos a los usuarios o clientes.

A este fin, se configuran distintas figuras administrativas: fundaciones, empresas públicas, consorcios y, en algún caso reciente, concesión administrativa, que permiten mayor autonomía, dinamismo y agilidad, introduciéndose en lo que se denomina «competencia gestionada».

Señalan los expertos como aspectos comunes de estos procesos, la importancia de la autonomía de los hospitales y de otros proveedores sanitarios, el desarrollo de la gestión clínica, la orientación de los servicios hacia la calidad, la recuperación del protagonismo de los usuarios, y el énfasis creciente en la efectividad de la práctica clínica y la medicina basada en la evidencia.

Como primer paso se pusieron en marcha los contratos-programas o contratos de gestión, los acuerdos entre los centros y el ente gestor con el fin de lograr el cumplimiento de los objetivos asistenciales. En el sector hospitalario en el ámbito del INSALUD, se introdujo la Unidad Ponderada Asistencial (UPA), y otras de naturaleza similar (UBA, EVA) en otras entidades gestoras, como medidas de la actividad hospitalaria.

El objetivo es ir aplicando sistemas de medición que caractericen mejor la producción hospitalaria, basados en la combinación de los casos tratados (case mix), grupos relacionados por el diagnóstico (GRD), categorías de gestión de pacientes (PMC), etc. 
Para poder avanzar en este sentido, es preciso disponer de una información básica sobre los diagnósticos y procedimientos, como la que ofrece el Conjunto Mínimo Básico de Datos (CMBD), junto a una adecuada contabilidad analítica.

Aunque aduciendo razones de urgencia, ya se había adelantado el INSALUD con el Decreto-Ley en el año 1996 y con la Ley 1997 de 15 de abril, (sobre habilitación de nuevas formas de gestión del Sistema Nacional de Salud y el Acuerdo de la Subcomisión Parlamentaria para la Consolidación y Modernización del Sistema Nacional de Salud). Aconsejan medidas de gestión que ya algunas CC.AA habían ido poniendo en marcha al amparo de su legislación específica, con la posibilidad de gestionar los servicios sanitarios del Sistema Nacional de Salud «directa o indirectamente a través de la constitución de cualesquiera entidades de naturaleza o titularidad pública admitidas en Derecho».

La creación de las Fundaciones Públicas Sanitarias en el ámbito del INSALUD, de acuerdo con lo expuesto sobre este asunto por el titular del Ministerio de Sanidad y Consumo en la Comisión del Senado del 10 de diciembre de 1998, "están orientadas a la mejora de la gestión y la eficiencia del sistema sanitario público a través de nuevas formas de gestión que posibiliten una verdadera y real autonomía de gestión en los centros sanitarios y, por ende, a garantizar la salvaguardia del propio sistema».

En la referida intervención, se señaló que ya diversas Comunidades Autónomas habían creado entidades singulares -los entes públicos sujetos al Derecho Privado en el País Vasco, la transformación del Sistema Catalán de Salud en un ente público de naturaleza institucional- y a configurar de un modo variado sus centros públicos.

Se destacó asimismo que Andalucía dispone de una fundación y tres empresas públicas, Canarias de una empresa pública, Cataluña de cuatro fundaciones, nueve empresas públicas y once consorcios. Galicia de cinco fundaciones y una empresa pública, el País Vasco dos empresas públicas; hay una fundación en Asturias y otra en Murcia, una empresa pública en Baleares y tres consorcios en Castilla León.

De hecho en mayo de 1999 existían en funcionamiento 14 fundaciones hospitalarias, 17 empresas públicas, 17 consorcios y una concesión administrativa.

Las características de las fundaciones públicas sanitarias, como pone en evidencia el Art. 111 de la Ley de Acompañamiento a los Presupuestos Generales del Estado para el año 1999, tratan de establecer una personificación jurídico-política adecuada a las circunstancias de las instituciones sanitarias públicas existentes, que garanticen especialmente el régimen estatutario del personal. 
Recientemente se ha publicado el Real Decreto 29/2000, de 14 de enero, sobre nuevas formas de gestión del Instituto Nacional de la Salud, que tiene como objeto el desarrollo reglamentario de las referidas Ley 15/1997 y artículo 111 de la Ley 50/98, que será de aplicación a los centros, servicios y establecimientos sanitarios de protección de la salud o de atención sanitaria gestionados por el Instituto Nacional de la Salud, que adopten cualquier tipo de gestión a través de fundaciones, constituidas al amparo de la Ley 30/1994, consorcios, sociedades estatales y fundaciones públicas sanitarias.

Llama la atención como últimamente los distintos Servicios Salud, prosiguen extendiendo la gestión clínica tanto en la asistencia especializada como en la primaria, bajo las formas de unidades de gestión o institutos.

Como resumen y en términos generales se puede señalar que existe un consenso ampliamente extendido, que la salud en España y estado de nuestros centros y servicios sanitarios, sigue siendo buena en términos generales, si lo comparamos con el que cabría esperar de nuestro nivel económico y de desarrollo. La posibilidad de acceso a los servicios en condiciones de igualdad y gratuidad se extiende a prácticamente a toda la población, con un amplio catálogo de prestaciones.

$\mathrm{El}$ aumento de la tasa de cobertura; el envejecimiento de la población con el incremento de la esperanza de vida; los avances tecnológicos tanto en el equipamiento como en la práctica médica y los nuevos medicamentos; la mejoría en lo que convencionalmente se denomina «prestación real media»-cantidad y calidad de los servicios que presta el sistema-; la evolución de los costes de los factores y la productividad de las organizaciones sanitarias; los cambios en el patrón de morbilidad de los ancianos con incapacidades funcionales y de personas mayores dependientes, obviamente demandarán de forma progresiva, una mayor preocupación y aportación presupuestaria para este gasto social -sanitario y cuidados sociales-, que determinará sustanciales cambios en la organización, ordenación, funcionamiento, tipos, distribución y dependencia de los centros sanitarios en el futuro.

\section{Bibliografía}

1 Comparecencia del Ministro de Sanidad y Consumo. Comisión del Senado. Boletín de las Cortes. 10 de diciembre de 1998.

2 CONSEJO ASESOR DE SANIDAD. Nuevas Tendencias en Organización Hospitalaria. Documento de Estudio 1997.

3 Encuesta Nacional de Salud de España 1997. Ministerio de Sanidad y Consumo. 1999. 


\section{Los últimos 20 años de los Centros Sanitarios en España}

4 Estadísticas de Establecimientos Sanitarios con Régimen de Internado de los años 1981, 1985, 1990 y 1995. Ministerio de Sanidad y Consumo e INE.

5 Fernández-Cuenca Gómez, R. Análisis de los Servicios Sanitarios. Informe SESPAS 1998. Escuela Andaluza de Salud Pública. Granada 1998.

6 Informe sobre la salud de los Españoles: 1998. Ministerio de Sanidad y Consumo. Madrid 1999.

7 Subsecretaría de la Seguridad Social. Libro Blanco de la Seguridad Social. Ministerio de Trabajo. Madrid 1977.

8 MARTÍN MARTíN, J.J. Cambio e innovación institucional. Las nuevas experiencias en gestión sanitaria. Informe SESPAS 1998. Escuela Andaluza de Salud Pública, Granada 1998.

9 Pemán Gavín. J. Derecho a la Salud y Administración Sanitaria. Publicaciones del Real Colegio de España de Bolonia. Cometa S.A. Zaragoza 1999.

10 Rey DE Castillo, J. Descentralización de los Servicios Sanitarios. Aspectos generales y análisis del caso español. Escuela Andaluza de Salud Pública. Granada 1998.

11 Rey del CASTillo, J. El futuro de la Sanidad Española. Un proyecto de reforma. Exlibris Ediciones S.L. Madrid 1999.

12 Segovia de Arana, J.M. La Sanidad en España. Documento de Estudio 1995.

13 Trovo Estaban, A.M y otros. Evolución del sector hospitalario 1972-1991. Revista Profesión Hospitalaria $n^{0}$ 4/1995.

14 TRUYoll WiNTRITH, I. La financiación de las Comunidades Autónomas: Presente y futuro. Revista de Administración Sanitaria, Vol III. Núm 12. octubre/diciembre 1999. 